\title{
Abstracts of Award-Winning Posters 11th Annual Health Sciences Poster Conference, Faculty of Medicine, Health Sciences Centre, Kuwait University, Kuwait, April 24-26, 2006
}

\section{Dr. Nael Al-Naqeeb Award for Best Undergraduate Student}

\section{1}

\section{Anxiety and Depression among Medical Doctors in} Kuwait: Prevalence and Associated Factors

H. Al-Mazeedi *, H. Al-Saleh, M. Al-Ajmi, A. Al-Rashed, M. Moussa

Department of Community Medicine, Kuwait University Faculty of Medicine, Kuwait

Introduction: To determine the prevalence of anxiety and depression among medical doctors specialized in internal medicine, surgery, obstetrics and gynecology, pediatrics, and anesthesia working in Kuwait's five general hospitals (Mubarak Al-Kabeer, Amiri, Adan, Farwania, and Jahra) and to investigate their association with sociodemographic characteristics, professional and work-related conditions, medical and family history, and life events. Methods: A cross-sectional survey was conducted in September 2005. The self-administered questionnaire included Hopkins Symptoms Checklist-25 as a psychological distress scale with total distress $>1.75$ as a cutoff to indicate psychological distress. Results: The prevalence of psychological distress was $24.3 \%$ among 310 respondents. Female doctors had a higher distress score than males, and age and distress levels were inversely related $(\mathrm{p}<0.001)$. Kuwaiti doctors had total distress twice that of non-Kuwaiti Arabs $(\mathrm{p}<0.001)$. Single doctors had higher distress scores than married doctors $(\mathrm{p}<0.001)$. Distress was highest among assistant registrars $(\mathrm{p}<0.001)$ and those in obstetrics and gynecology $(p=0.006)$. Doctors who sleep in hospital during night duties, experience frequent verbal or physical abuse, have respiratory or gastrointestinal diseases, and were diagnosed with depression had higher distress than those who did not. There was an inverse relationship between number of experience years and distress as well as between job satisfaction and the percentage of total distress. The number of major life events and distress levels were proportional. Conclusions: Psychological distress is prevalent among medical doctors and is associated with sociodemographic and work-related conditions. Our results invite the health authorities to take action to alleviate doctors' work burden, some of which may be caused by preventable factors. Kuwaiti doctors were found to have higher levels of anxiety and depression than non-Kuwaiti doctors.

\section{Best Postgraduate Student Awards}

\section{Basic Sciences (MSc)}

\author{
Estrogen Receptor Gene Silencing in Breast Cancer \\ Cells Using siRNA \\ A.A. Al-Azmi ${ }^{*}$, a, Y.A. Luqmani ${ }^{\text {b }}$, M.M. El-Zawahri a , G. Abraham ${ }^{\text {b }}$, \\ C.H.J. Ford ${ }^{\mathrm{C}}$, B. Al Bader ${ }^{\mathrm{d}}$ \\ aDepartment of Molecular Biology, Faculty of Science; \\ ${ }^{b}$ Department of Pharmaceutical Chemistry, Faculty of \\ Pharmacy; Departments of 'Surgery and 'Physiology, \\ Faculty of Medicine, Kuwait University, Kuwait
}

Introduction: Inhibition of estrogen receptor $\alpha(\mathrm{ER} \alpha)$ is one of the major strategies for prevention and treatment of breast cancer. However, failure to overcome development of endocrine resistance, arising despite continued expression of tumour ER $\alpha$, limits this approach. Our aim was to produce a model system to inves-

\section{KARGER}

Fax +41613061234 E-Mail karger@karger.ch www.karger.com
(C) 2006 S. Karger AG, Basel

$1011-7571 / 06 / 0156-0463 \$ 23.50 / 0$

Accessible online at: www.karger.com/mpp 
tigate cellular consequences of loss of function of $\mathrm{ER} \alpha$, by establishing a breast cancer cell line with a permanent source of small interference RNA (siRNA) to specifically inhibit production of ER $\alpha$ protein. Methods: Three siRNA constructs (pI-III), targeting various sequences of human $\mathrm{ER} \alpha$, and a scrambled sequence were cloned into the pRNA-U6.1/Neo GenScript vector. MCF7 breast cancer cells were transformed with 2 or $4 \mu \mathrm{g}$ of each plasmid (6 and 24 h exposure), using Lipofectin or Xtreme reagent conjugates. Transformants were rescued by growth in G418 selection medium. ER $\alpha$ mRNA levels were determined by real-time RT-PCR of extracted RNA, and ER $\alpha$ protein by Western blotting; normalisation was achieved by simultaneous analysis of $\beta$-actin. Presence of plasmid DNA in transformants was verified with primers targeting various regions of the vector. Results: Stably transfected cells maintaining antibiotic resistance over several passages were established by continuous culture. Linearised antiER $\alpha$-siRNA construct pII most effectively down-regulated ER $\alpha$ (as evidenced by mRNA and protein analysis) in these cells as compared to G418-resistant transformants containing scrambled siRNA; complete knockdown was not observed. Whereas $4 \mu \mathrm{g}$ produced more transformants, 24-hour exposure did not increase transformation efficiency. Conclusions: Using ER $\alpha$ siRNA vector constructs, we have successfully established a long-term culture of MCF7 breast cancer cells that exhibit decreased expression of $\mathrm{ER} \alpha$. This is hoped to provide a model system in which to study aspects of endocrine resistance. Funding agency: Kuwait University College of Graduate Studies, grant No. YS01/04.

\section{Basic Sciences (PhD)}

\section{Identification of Species-Specific and Major Antigenic Proteins of Mycobacterium tuberculosis to Replace Tuberculin in the Diagnosis of Tuberculosis S.N.M. Hanif*, R. Al-Attiyah, A.S. Mustafa}

Department of Microbiology, Faculty of Medicine, Kuwait University, Kuwait

Introduction: Tuberculosis (TB) is a major global health threat with 8-10 million people developing active disease and 2-3 million dying of TB annually. Although tuberculin is widely used to detect infection with Mycobacterium tuberculosis, its diagnostic efficacy is limited due to antigenic cross-reactivity with the BCG vaccine and environmental mycobacteria, e.g. Mycobacterium avium and Mycobacterium vaccae etc. The aim of this study was to identify $M$. tuberculosis-specific proteins to replace tuberculin in diagnostic applications. Methods: DNA corresponding to five major antigenic proteins of $M$. tuberculosis, i.e. PE35, PPE68, CFP10, ESAT6 and Rv3619c, was PCR-amplified from the genomic DNA of M. tuberculosis, and cloned and expressed in Escherichia coli, and the expressed proteins were purified by affinity chromatography. Antigen-specific antibodies, raised against the purified proteins in rabbits, and reverse transcriptase-PCR were used to detect gene expression in mycobacteria at protein and
mRNA levels, respectively. The diagnostic potential of the proteins was investigated by studying tuberculin-type delayed type hypersensitivity (DTH) responses in guinea pigs infected with $M$. tuberculosis, BCG, M. avium and M. vaccae. Results: All of the five proteins (PE35, PPE68, CFP10, ESAT6 and Rv3619c) were expressed at protein and mRNA levels in $M$. tuberculosis, but none of them were expressed in M. avium and M. vaccae, and only Rv3619c was expressed in BCG. The studies in guinea pigs showed that PE35, PPE68, CFP10, ESAT6 and Rv3619c proteins elicited positive DTH responses in $M$. tuberculosis-infected but not in $M$. avium- and $M$. vaccae-infected animals, whereas only Rv3619c elicited positive DTH responses in BCG-infected guinea pigs. Conclusions: PE35, PPE68, CFP10, ESAT6 proteins are specific to $M$. tuberculosis and induce positive DTH responses only after infection with $M$. tuberculosis. These proteins could therefore replace tuberculin in order to discriminate between infection with $M$. tuberculosis, BCG vaccination and exposure to environmental mycobacteria. Funding agency: Kuwait University Research Administration, Project No. YM01/03.

\section{Resident}

\section{Reliability of Gingival Bleeding on Probing and Periodontal Probing Depth in Patients with Plaque-Induced Gingivitis}

\author{
F. Al-Aradi, D. Boodai
}

Kuwait University Faculty of Dentistry, Kuwait

Introduction: To assess the reliability of bleeding on probing (BOP) and periodontal probing depth (PD) after repeated probing at a time interval of $4 \mathrm{~h}$ between re-examination procedures in patients with plaque-induced gingivitis. Methods: Fifteen nonsmoking adult volunteers, 18-25 years of age, with plaque-induced gingivitis participated. Full mouth examination was performed twice for the same subjects. Periodontal probing depth (PD1/mm and PD2/mm), bleeding on probing (BOP1 and BOP2), and bleeding index (BI1 and BI2) were assessed at six sites of each tooth present. Probing was repeated $4 \mathrm{~h}$ (T2) after the first probing (T1). Intra- and interexaminer patterns of re-examination were considered. Results: When ignoring the re-examination pattern, subject-specific analysis revealed mean differences of $0.061,-0.005$, and -0.003 for PD, BI, and BOP, respectively. In addition, kappa statistics were employed for site-specific analysis, in which the highest kappa value of 0.481 was found when assessing PD. Thorough subject-specific analysis of intra-examiner agreement for both examiners showed mean differences close to zero for all three variables. Fair kappa values of 0.544 and 0.465 were obtained from site-specific analysis of PD for E1 and E2, respectively, while poor values were resulted for both BI and BOP. When inter-examiner pattern of re-examination was considered, analysis of agreement revealed mean differences close to zero for all variables. Kappa values were found to be fair for PD, but poor for both BI and BOP. Conclusions: It is concluded that no significant 
difference between inter- and intra-examiner reliability in the assessment of $\mathrm{PD}, \mathrm{BI}$, and $\mathrm{BOP}$ was found. In general, the assessment of PD showed to have fair agreement, while that of BI and BOP was poor.

\section{Basic and Applied Sciences Awards}

\section{1 \\ Novel Arylcarbonyl- and Arylsulfonyl-Piperazinyl Oxazolidinones with Antibacterial Activity}

\author{
O.A. Phillips *, a, E.E. Udo b ${ }^{\text {, A.A.M. Alic }}$, S. Samuel ${ }^{\mathrm{a}}$
}

aDepartment of Pharmaceutical Chemistry, Faculty of

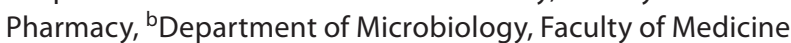
and 'Department of Chemistry, Faculty of Science, Kuwait University, Kuwait

Introduction: The bio-isosteric replacement of the 5-acetamidomethyl group in linezolid with the triazolylmethyl moiety gave $\mathrm{PH}-027$, a novel 5-triazolylmethyl oxazolidiNones with improved antibacterial activity against drug-resistant Gram-positive bacteria. The aims of our study were to synthesize a series of novel arylcarbonyl- and arylsulfonyl-piperazinyl 5-triazolylmethyl oxazolidiNones $5 \mathrm{a}-\mathrm{u}$, evaluate their antibacterial activity and correlate this to the calculated log of partition coefficient (ClogP) values. Methods: A series of novel oxazolidiNones were synthesized and their antibacterial activity against Gram-positive clinical isolates evaluated in comparison to linezolid, vancomycin and $\mathrm{PH}-027$. The organisms tested $(\mathrm{n}=49)$ included methicillin-resistant $(\mathrm{n}=10)$ and -susceptible Staphylococcus aureus $(\mathrm{n}=11)$; methicillin-resistant $(n=3)$ and -susceptible coagulase-negative staphylococci $(\mathrm{n}=8)$; Streptococcus pneumoniae $(\mathrm{n}=6)$, and vancomycin-resistant (VRE, $\mathrm{n}=4$ ) and -susceptible (VSE, $\mathrm{n}=7$ ) enterococci. Minimum inhibitory concentrations (MICs, $\mu \mathrm{g} / \mathrm{ml}$ ) were determined by agar dilution method on Mueller-Hinton agar, in the absence and presence of $50 \%$ human plasma, with the medium containing dilutions of antibacterial agents ranging from 0.12 to 32 or $64 \mu \mathrm{g} / \mathrm{ml}$. ClogP values were computed using CS ChemDraw Ultra software. Results: Against staphylococcal strains, the arylcarbonyl-oxazolidiNones 5a-p (ClogP: 0.0461.66) showed strong antibacterial activity (MIC value range $0.12-$ $2 \mu \mathrm{g} / \mathrm{ml}$ ). The arylsulfonyl derivatives $5 \mathrm{r}-\mathrm{t}$ with $\mathrm{Clog} \mathrm{P}$ values $>1.70$ were generally less active $(2->32 \mu \mathrm{g} / \mathrm{ml})$; while the benzenesulfonyl $5 \mathrm{q}(0.5-2 \mu \mathrm{g} / \mathrm{ml})$ and thienylsulfonyl $5 \mathrm{u}(0.5-1 \mu \mathrm{g} /$ $\mathrm{ml})$ derivatives with $\mathrm{Clog} \mathrm{P}<1.70$ were more active. The 3 -methoxyphenyl 5j, 3-nitrophenyl 5m, 2-thienyl 50 and 2-furyl-carbonyl 5 p derivatives showed excellent activity against $S$. pneumoniae $(0.25-0.5 \mu \mathrm{g} / \mathrm{ml})$. The arylcarbonyl derivatives $5 \mathrm{a}-\mathrm{p}$ showed strong activity against VSE and VRE strains $(0.5-2 \mu \mathrm{g} /$ $\mathrm{ml})$. Some of the compounds showed significantly increased MICs in the presence of $50 \%$ human plasma, suggesting plasma instability or binding. Conclusions: Arylcarbonyl oxazolidiNones showed activity superior to the arylsulfonyl derivatives, and they were also superior or comparable to linezolid, vancomycin and PH027. Finally, significant structure-antibacterial activity relationships are highlighted. Funding agency: Kuwait University Research Administration, Project No. PCO1/02.

\section{2 \\ Diabetes-Induced Activation of Peroxisomal Functions in Kidneys of Spontaneously Hypertensive Rats Is Ras-GTPase-Mediated}

G.S. Dhaunsi ${ }^{\text {a }}$ M.H. Yousif ${ }^{\text {b }}$, M. Alsaeid ${ }^{\text {a }}$, I.F. Benter ${ }^{\text {b }}$

Departments of aPediatrics and bPharmacology, Faculty of Medicine, Kuwait University, Kuwait

Introduction: Peroxisomes and peroxisome proliferator-activated receptors (PPAR) are known to contribute towards the pathogenesis of diabetes and hypertension. The objective of this study was to examine the role of Ras-GTPase in the activation of peroxisomal functions in kidneys of diabetic and/or hypertensive rats. Methods: Diabetes was induced in male Wistar-Kyoto (WKY) rats and spontaneously hypertensive rats (SHR) by streptozotocin (STZ) and animals were divided into eight groups $(\mathrm{n}=$ 4) for treatment with FPT-III (Ras-GTPase inhibitor). Activities of peroxisomal enzymes, catalase, acyl-CoA oxidase and $\beta$-oxidation of lignoceric acid were assayed in kidney homogenates. Western blotting was done to detect PPAR- $\gamma$, peroxisome membrane protein (PMP-70) and catalase. Results: STZ decreased $(\mathrm{p}<0.01)$ catalase activity in kidneys from normotensive and hypertensive rats, however, catalase protein was significantly (1.5fold) increased. Treatment of normotensive or hypertensive diabetic rats with FPT-III prevented the inhibitory effects of diabetes on catalase enzyme activity. Acyl-CoA oxidase activity and oxidation of lignoceric acid were significantly $(\mathrm{p}<0.01)$ increased following STZ injection in SHR and WKY rats. FPT-III treatment significantly $(\mathrm{p}<0.01)$ decreased activities of acyl-CoA oxidase and lignoceric acid oxidation only in tissues from SHR. Diabetes increased (3-fold) PMP-70 protein in both SHR and WKY rats, however, FPT-III did not affect diabetes-induced PMP-70 expression in SHR or WKY rats. PPAR- $\gamma$ was not markedly affected by diabetes in SHR or WKY rats. FPT-III increased (2-fold) the expression of PPAR- $\gamma$ in SHR and WKY rats. Conclusions: Increase in the expression of PMP-70 and catalase, and activities of acylCoA oxidase and lignoceric acid oxidation indicate peroxisome proliferation as a result of STZ-induced diabetes in SHR and WKY rats. FPT-III-mediated effects on peroxisomal functions and PPAR- $\gamma$ in kidneys from SHR or WKY rats suggest a role for Ras-GTPase signaling pathways in the pathogenesis of diabetes and/or hypertension. Funding agency: Kuwait University Research Administration, Project No. RM02/03. 


\section{Clinical Science Awards}

1

\section{A Rapid and Sensitive Method for the Diagnosis of the 'Avian Flu' H5 Subtype of Influenza A Virus in Kuwait}

\author{
S. Loutfy*, N. Madi, D. Khalik, W. Chehadeh
}

WHO Collaborating Center for Acquired Immunodeficiency Syndrome (AIDS) and WHO Collaborating Center for Virus Reference and Research for the Eastern Mediterranean Region (EMR), Virology Unit, Department of Microbiology, Faculty of Medicine, Kuwait University

Introduction: The highly pathogenic avian influenza (H5N1) virus 'bird flu' continues to pose a serious global human public health risk and the potential for a pandemic remains great. Therefore, the need for a rapid and sensitive method to diagnose the infection, subtype the virus and measure the quantity of the virus in respiratory and other samples (which predicts the potential severity and seriousness of the disease) is of paramount importance in the management of this pandemic. Methods: We have developed a sensitive and specific multiplex RT-PCR capable of detecting common respiratory viruses causing acute respiratory disease in Kuwait. Those found positive for influenza A virus were then subjected to a quantitative real-time PCR to subtype the virus as $\mathrm{H} 5$ and to measure the amount of virus in respiratory samples. A 189-bp fragment of the influenza virus A H5 gene was amplified with specific primers and quantitatively detected with probes supplied in the LightMix (TIB MOLBIOL, Berlin). The supplied standard dilutions of influenza A $\mathrm{H} 5$ virus cDNA ranging from 10 to 1 million copies $/ \mathrm{ml}$ allow the absolute quantification of the viral cDNA in unknown samples. Results: A standard curve using standard increasing dilutions of influenza A H5 was plotted to quantitate influenza A virus of the $\mathrm{H} 5$ subtype. The minimum detection limit was 10 copies $/ \mathrm{ml}$. Specificity of the primer/probe mixture was tested on samples containing rhinovirus, coronavirus, respiratory syncytial virus, measles and mumps viruses. Out of the 10 of $80(12.5 \%)$ patients with acute respiratory disease that were influenza virus A-positive, none was found to be of the $\mathrm{H} 5$ subtype of influenza A virus so far in Kuwait. Conclusions: The combination of the multiplex RT-PCR developed in our laboratory and the LightMix system used in subtyping and quantitation of influenza A virus of the $\mathrm{H} 5$ subtype is a rapid and sensitive assay for the rapid diagnosis of influenza A H5 subtype. Funding agency: Kuwait University Research Administration, Project No. M1 01/04.

\section{2}

Soluble Transferrin Receptor, Transferrin Receptor, Ferritin Index, and Iron Status in Female Patients with Type 2 Diabetes

O.A.Mojiminiyi ${ }^{\text {a }}$, N.A. Abdella ${ }^{b}$, R. Marouf ${ }^{a}$, S. George ${ }^{\mathrm{a}}$, C. Pinto ${ }^{\mathrm{b}}$, R. Mathew ${ }^{\text {a }}$

Departments of a Pathology and ${ }^{\mathrm{b}}$ Medicine, Kuwait University Faculty of Medicine, Kuwait

Introduction: Anaemia is a common finding in type 2 diabetic (T2DM) patients. Diagnosis of iron deficiency anaemia (IDA) in diabetic patients is difficult because of complications which cause anaemia with high ferritin levels (as in anaemia of chronic disease, ACD), even in the presence of iron deficiency. Soluble transferrin receptor (sTfR), derived primarily from erythroid progenitor cells in the bone marrow, has emerged as a reliable index for the integrated effects of iron reserve, iron availability, and erythropoietic stimulation. The aim of this study was to evaluate iron metabolism parameters (ferritin, iron, transferrin and sTfR) and erythropoiesis-stimulating factor (serum erythropoietin, Epo) in assessing anaemia in female patients with type 2 diabetes. Methods: High-sensitivity C-reactive protein (hs-CRP), full blood count, Epo, sTfR, transferrin, transferring saturation, ferritin, and iron were determined in 96 female patients with T2DM. Patients were classified as anaemic $(\mathrm{Hb}<12 \mathrm{~g} / \mathrm{dl})$ or normal and the sTfR/log ferritin ratio (sTfR-F index) $>0.8$ was used as a marker for identification of iron-deficient erythropoiesis. Results: 18 (18.8\%) patients were anaemic and all had either IDA or were classified as having ACD. hs-CRP was not significantly different between the normal [median (range) $=0.50(0.1-5.0 \mathrm{mg} / \mathrm{l})$ ] and anaemic [median (range) $=0.30(0.1-2.1 \mathrm{mg} / \mathrm{l})]$ groups. Of the anaemic patients, $44 \%$ had IDA and $56 \%$ had ACD and, among the latter group, $70 \%$ showed evidence of a defective endogenous Epo production $($ Epo $<5 \mathrm{mU} / \mathrm{ml})$ and $50 \%$ had sTfR-F index $>0.8$. Regardless of the cause of the anaemia, sTFR $>1.7 \mu \mathrm{g} / \mathrm{ml}$ and sTfR-F index $>0.9$ correctly identified anaemic patients with $100 \%$ sensitivity. Conclusions: The majority of anaemic female patients with T2DM have ACD with low Epo and/or iron-deficient erythropoiesis. sTfR and sTfR-F index could be used as tools for diagnosis and selection of therapeutic modality in diabetic patients with anaemia. Funding agency: Kuwait Foundation for Advancement for Sciences, Grant No. 2004-07-02. 


\section{3}

\section{Antibodies to pp150 and pp28 Cytomegalovirus Antigens Protect Patients from Infection and Disease after Kidney Transplantation}

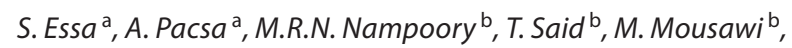
W. Al-Nakib a

${ }^{a}$ Department of Microbiology, Faculty of Medicine, Kuwait

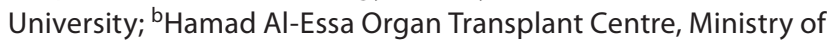
Health, Kuwait

Introduction: One of the most important and common complications following organ transplantation is infection with cytomegalovirus (CMV). The immunological factors that confer protection against infection and disease due to CMV are not well characterized. This study aims at identifying antibodies to those antigens which contribute to the protection against CMV infection and/or disease. Methods: In this study 77 kidney recipients (KR) were enrolled. All had CMV antibody due to previous infection. The virus is most probably present latently in their body. However, the nature and type of antibody to CMV are not known. KR were followed up for a period of 6 months after transplantation for the development of active CMV infections by the antigenemia assay. The antibody responses before and after transplantation to $5 \mathrm{CMV}$-related peptide antigens (pp65, gB, pp150, pp28 and pp38) were investigated by ELISA and the results were correlated with the development of CMV infections/disease. Results: During the follow-up period 23 (30\%) of the $77 \mathrm{KR}$ had an active $\mathrm{CMV}$ infection. Among these CMV-infected KR, there was only 1 patient who had antibodies to both pp150 and pp28 antigens. In contrast, 26 of 54 (48\%) KR who did not develop CMV infection/ disease had such antibodies $(\mathrm{p}<0.0001)$. There was, however, no significant difference in the antibody responses to the other three CMV peptide antigens between the infected and noninfected KR $(p>0.05)$. Conclusions: The findings of this study suggest that antibodies against pp150 and pp28 antigens may play an important and protective role against the reactivation (and probably primary infection) of CMV infections after transplantation. These results also suggest that patients lacking antibodies to pp150 and pp28 prior to transplantation are probably at 'high risk' of developing CMV infection and hence have to be closely monitored. Funding agency: Kuwait University Research Administration, Project No. MI 03/02.

\section{Case Report Award}

\author{
1 \\ Congenital Heart Disease, Cerebral Palsy, \\ Developmental Delay, and Gastrointestinal \\ Abnormalities: A New Syndrome? Case Report and \\ Literature Review
}

T.B. Al-Fahad

Department of Medicine, Mubarak Al Kabeer Hospital, Kuwait

Background: We observed the unusual association of congenital heart defects, cerebral palsy, developmental delay, epilepsy and gastrointestinal abnormalities with a unique chromosomal abnormality. Case Summary: A girl was born after an uneventful pregnancy and term delivery to related healthy parents of Kuwaiti origin. She was normal until 2 months of age when she presented to our hospital with shortness of breath, cyanosis and vomiting. Physical examination was significant for microcephaly $(\mathrm{HC}<5$ th percentile), large ears, and ejection systolic and diastolic murmurs. Abnormalities of the nervous system included: mixed type cerebral palsy, epilepsy (developed later), developmental delay, and no response to visual or auditory stimuli. Echocardiography showed very large ASD, large VSD, moderately large PDA, and dilatation of both left atrium and ventricle. She was in severe heart failure and pulmonary hypertension requiring prolonged ventilation. CT brain scan showed diffuse cerebral atrophy and multiple areas of infarction. MRI findings were compatible with hypoxic ischemic encephalopathy, calcifications of cortex, and severe global atrophy. Gastrointestinal abnormalities were limited to gastroesophageal reflux disease, gastroparesis and impaired gag reflex. Fundus examination was normal with decreased blood vessels. Chromosomal analysis showed 46XX der8 (10qter-10q21:: $8 \mathrm{p} 21-8 \mathrm{qter})$ in the patient, while in the mother 46XX t $(8 \mathrm{p} ; 10 \mathrm{q})$ (10qter-10q22:8p21-8qter,10pter-10q22:8p21-8pter). The father had normal 46XY and both parents were phenotypically normal. Conclusion: This is a new syndrome in terms of both constellation of symptoms and the genetic abnormality. Presented is a literature review of the symptoms and the genetic abnormality. 